\title{
Deep Learning Neural Network Prediction System Enhanced with Best Window Size in Sliding Window Algorithm for Predicting Domestic Power Consumption in a Residential Building
}

\author{
Dimpal Tomar $\mathbb{D}^{1},{ }^{1}$ Pradeep Tomar $\mathbb{D}^{1},{ }^{1}$ Arpit Bhardwaj $\mathbb{D}^{2},{ }^{2}$ and G. R. Sinha $\mathbb{D}^{3}$ \\ ${ }^{1}$ Gautam Buddha University, Greater Noida, UP, India \\ ${ }^{2}$ BML Munjal University, Gurugram, Haryana, India \\ ${ }^{3}$ MIIT Mandalay, Mandalay, Myanmar
}

Correspondence should be addressed to G. R. Sinha; gr_sinha@miit.edu.mm

Received 29 December 2021; Accepted 29 January 2022; Published 2 March 2022

Academic Editor: Vijay Kumar

Copyright $\odot 2022$ Dimpal Tomar et al. This is an open access article distributed under the Creative Commons Attribution License, which permits unrestricted use, distribution, and reproduction in any medium, provided the original work is properly cited.

Buildings are considered to be one of the world's largest consumers of energy. The productive utilization of energy will spare the accessible energy assets for the following ages. In this paper, we analyze and predict the domestic electric power consumption of a single residential building, implementing deep learning approach (LSTM and CNN). In these models, a novel feature is proposed, the "best $\mathrm{N}$ window size" that will focus on identifying the reliable time period in the past data, which yields an optimal prediction model for domestic energy consumption known as deep learning recurrent neural network prediction system with improved sliding window algorithm. The proposed prediction system is tuned to achieve high accuracy based on various hyperparameters. This work performs a comparative study of different variations of the deep learning model and records the best Root Mean Square Error value compared to other learning models for the benchmark energy consumption dataset.

\section{Introduction}

Today's modern world faces steady acceleration in the development of technology, population, and economic growth, which dramatically increases energy consumption. As per the International Energy Agency (IEA) report, more than $30 \%$ of the global energy is consumed in buildings, and nearly one-third of carbon dioxide $\left(\mathrm{CO}_{2}\right)$ emitted by the building accounts for a significant part of the total $\mathrm{CO}_{2}$ emissions [1]. Hence, buildings are the world's prime consumers of energy, and broadly, in residential sectors, heating and cooling systems consume energy, accounting for more than one-fifth of the total [2]. Though being a good consumer of energy, buildings are also connected with a significant proportion of energy waste as well [3], and this dissipated form of energy shows an alarming situation to sustainability. Such frightening circumstances address the concerns of growing energy demand, development of urbanization, and pollutant emissions, the increasing need of new smart sustainable energy resources. Hence, it is necessary to emerge with solutions that deal with building energy efficiently as it is extremely crucial. With this pattern, accurate predictions of future electric power consumption have become a fundamental advance in the computerized administration of power systems.

We can abate the further rise in the above-mentioned primary issues, namely, pollutant emissions (e.g., $\mathrm{CO}_{2}$ ) and energy consumption in buildings, only by promoting energy-saving designs for buildings during the design phase [4]. To accomplish smart and sustainable designs for energy efficiency, a focus has been derived towards the integration of buildings and smart technology for modeling and forecasting energy consumption. A smart environment is composed of "networks of sensors" that produce substantial amounts of energy data [5], for instance, the smart grid, the next level of the future power grid that dynamically deals with the generation and distribution of energy; therefore, at aggregate as well as modular level, smart, intelligent, and 
reliable decisions should be made endlessly in the grid with flexibility [6]. However, to achieve the reliability and efficiency of the grid, it is important to predict the future energy demand. In addition to that, smart meters are also quite promising in understanding the behavior of energy consumption with better clarity to the consumers as they do not save energy by themselves and are capable of sending precise readings to the power suppliers for customer billing. However, during the past decades, various countries like the United States of America (USA), China, and Europe have been working on energy efficiency by conducting programs of deploying smart meters at a large scale [7-10]. This smart technology will benefit in lowering electricity bills and optimizing power consumption, which will play a significant role in the development of sustainable smart cities and societies. But, we should take into account that innovation and technology all alone will not be sufficient to change how individuals devour energy, yet it gives the intention to utilize energy in a purposeful and cognizant manner. Furthermore, resident's behavior and households count are the secondary factors having a substantial influence on the energy efficiency profile of domestic buildings. Particularly, the occupancy of various household appliances is primarily responsible for obtaining the household electric power consumption. Beckel, Sadamori and Staake [11] reveal characteristics of the households, number of occupants, and appliances using energy-related data generated from smart meters. Haldi and Robinson [12] tried to predict the impact of occupant's behavior and their presence on the energy demand in buildings.

Artificial intelligence (AI) techniques have been applied in a wide range of disciplines and attracted the attention of researchers or scientists. AI models perform powerful data modeling by simulating the obtained information and adapt to changes in historical data to infer new facts [13]. Various qualitative works have recognized that AI techniques like statistical and learning-based modeling are ample skillful to apprehend nonlinear and tricky relationships that yield extremely good accuracy estimates from the historical energy-related data of buildings [13-15]. These AI models are quite promising for the prediction of domestic energy consumption in residential buildings accurately. However, it widely depends upon the researcher's requirement and their work on how to derive these AI models. Also, the success of AI techniques usually relies upon "data representation." Out of them, deep learning aspires to form a combination of various nonlinear transformations and helpful interpretation in a more abstractive way and ultimately achieve more benefit [16]. Hence, the deep learning approach has been vigorously considered in different application areas such as image and video recognition [17-20], speech recognition $[21,22]$, and natural language processing $[23,24]$.

The deep recurrent neural networks are powerful tools dealing with the long sequence modeling in various domains of time series like energy demand and usage prediction. This work chooses Long Short-Term Memory (LSTM) and Convolution Neural Network (CNN) with an enhanced sliding window algorithm for predicting domestic energy consumption. The advantages of this enhanced deep learning model include (1) capturing the nonlinear correlation over multivariable energy data composed of exogenous and target variables, (2) modeling the temporal information into separable spaces for energy predictions, (3) identifying the best " $N$ " window size, that is, the number of days considerable in the past that yields the optimal models followed by the best $\mathrm{N}$ window size graph for both the deep learning models, and (4) hyperparameter tuning of the models for more accurate predictions.

1.1. Related Work. Several studies have been devoted to predicting energy consumption at an industrial and residential level. Analyzing energy performance in buildings, which has been the subject of many researchers, is categorized into two modeling approaches: traditional statistical approach and machine learning approach. Table 1 presents the summary of researches conducted on energy consumption in buildings and their proposed method based on two categories, namely, conventional statistical methods and traditional machine learning modeling.

1.1.1. Conventional Statistical Methods. Conventional statistical methods are popular and classical modeling techniques that have been widely used to solve forecasting problems in energy consumption. Several popular statistical models for predicting energy consumption in buildings include autoregressive (AR) models such as AR, Autoregressive Integrated Moving Average (ARIMA), and variants of ARIMA model named Seasonal Autoregressive Integrated Moving Average (SARIMA). Other statistical regression algorithms are linear regression and multilinear regression, Bayesian regression, ordinary least square regression, and case-based reasoning.

AR model works on the fundamental of analyzing the statistical properties of energy-related time-series data. In other words, AR models predict future energy consumption only by considering the recent past and area data at a specific time. The implementation of these AR models was relatively simple and worked upon the theory of only looking back into the past data of dependent or target variables, but at the same time, these models have shown significant limitations, such that the forecasting horizon is limited to the short term only and cannot determine complex and nonlinear data patterns, which limit their application scope and accuracy [25-28]. However, researchers introduced several enhancements to overcome basic autoregressive models issues such as SARIMA models, Autoregressive Integrated Moving Average with Explanatory Variable (ARIMAX) models, and Seasonal Autoregressive Integrated Moving Average with Explanatory Variable (SARIMAX), but these improved versions of AR models are still facing some limitations accounting for not capturing nonlinear relationship within building energy-related time-series data, which highly affects the forecasting performance. In addition to these technical improvements, many researchers came up with the idea of hybrid models to tackle the above issues, such as ARIMA and Artificial Neural Network (ANN), ARIMA, and 
TABLE 1: Summary of researches conducted on energy consumption in buildings.

\begin{tabular}{lcccc}
\hline Technique & Focus of work & Sector and case study & Performance metrics & Pub. \\
\hline ARMA and ARIMA & $\begin{array}{c}\text { Analysis of household } \\
\text { electricity consumption }\end{array}$ & $\begin{array}{c}\text { French residential building } \\
\text { (energy consumption data) }\end{array}$ & $\begin{array}{c}\text { Akaike Information Criterion } \\
\text { (AIC); Root Mean Square Error } \\
\text { [25] }\end{array}$ \\
\hline ARIMA & Forecast the future demand & $\begin{array}{c}\text { Residential building (power } \\
\text { consumption) }\end{array}$ & Mean Square Error (MSE) \\
\hline [51]
\end{tabular}

Linear regression, feedforward neural network (FFNN), SVM, least-squares SVM, and hierarchical mixture of experts: regression and FFNN and fuzzy c-means

with FFNN

Predicting one hour ahead electric energy consumption and a short-term forecasting scenario
Residential buildings; Campbell Creek 3 homes dataset (electricity consumption)
Coefficient of Variance (CV), Mean Absolute Percentage Error (MAPE), and MBE
Random forest

Predicting average and peak energy consumption based on telecommunication data (electric people dynamics

Energy consumption

SVM

$$
\begin{aligned}
& \text { prediction based on weather } \\
& \text { and building operating } \\
& \text { parameters }
\end{aligned}
$$

Analysis and prediction of IoT-based sensor data in a building using different Multilayer perceptron, linear regression, random forest, and SVM

followed by a rigorous comparative study with other learning techniques
Mean Absolute Error (MAE), MSE, RMSE, Relative Squared Error (RSE), Relative Absolute Error (RAE), and coefficient of determination (R2)

Commercial building; 3-star

hotel energy dataset (weather

data, lighting data, elevator, and

MSE; R2 cooling system data)

Two-storey building (weather, light and appliances energy consumption, and temporal R2, MAPE, MAE, and RMSE information)

To predict long-term energy consumption based on an

Fuzzy Bayesian econometric methodology to improve reliability and accuracy

Ensemble bagging trees

Energy use prediction at hourly granularity

Institutional building; Rinker hall

data (climatic, occupancy and temporal data)

Chinese per capita electricity consumption (PEC) dataset

MAE, MAPE, and RMSE (electricity dataset)

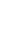

hourly granularity

\begin{tabular}{l} 
LSTM \\
\hline Conditional Restricted \\
Boltzmann Machine (CRBM) \\
and Factored Conditional \\
Restricted Boltzmann \\
Machine (FCRBM) \\
CNN-LSTM with fixed \\
window size
\end{tabular}

Forecasting energy load with one min and one-hour time resolution

Residential building; single house data (power consumption)

R2, RMSE, and MAPE

The energy consumption

forecasting over different time Residential building; household horizons (short, medium and long term) and on different electric dataset (energy consumption)

RMSE, $\mathrm{R}$, and $p$-value time resolutions

Prediction of electricity
consumption for next hour

Residential building; (electricity consumption dataset)
RMSE 
evolutionary models and collaboration with other machine learning algorithms [29-32].

On the other hand, statistical regression algorithms work on modeling the relationship between predictor and target variables. Various researchers predicted energy consumption in buildings by using linear regression [33-35], multiple regression [36, 37], and Bayesian regression [38]. Regression models suffer from multicollinearity problems that arise due to the relationship among independent variables used in prediction. Additionally, it is difficult to determine the explanatory variables, and we are still facing difficulty in dealing with the nonlinear problems.

\subsubsection{Traditional Machine Learning Modeling. Machine} learning modeling acts as a black box and tries to learn the relationship that exists among input features and targets based on the provided data, such as energy performance. Several machine learning algorithms have been studied by researchers in buildings for estimating the energy consumption, energy demand, and their related performance criteria for different circumstances. Numerous techniques are widely used to analyze energy consumption prediction problems in various types of buildings, that is, commercial, residential, or industrial sectors.

Being a highly adaptable and flexible approach, ANN solved numerous prediction problems with respect to energy consumption [39-42], and nearly all the related works revealed that ANN outperforms real-time problems and identifies the nonlinear relations between input and output but at the same time deals with the several issues like overfitting problems when additional energy feature is introduced and local minima problems.

Besides that, support vector machines (SVM) are another efficient and widely popular modeling approach for solving nonlinear problems accounting for efficient energy management in buildings by forecasting energy consumption which covers [43-48]. These learning algorithms maintain a balance between nonlinearity and prediction accuracy. Though users find difficulty in identifying the kernel functions, an optimal hyperparameter will produce the optimal accurate prediction model. Users need to do a lot of rigorous work on the dataset properties and their own experience to deal with such issues.

There are other learning modeling employed black-box approaches used for different objectives such as detection and diagnosis of faults and forecasting energy consumption at different horizons with respect to buildings, with the focus of performance improvement covering ensemble models and improved hybrid models $[49,50]$.

Kim and Cho [16] predicted residential energy using a hybrid approach of deep learning, which uses a sliding window algorithm with fixed window size. The window size also affects the model performance. Besides that, model needs hyperparameter optimization as these parameters have a great impact on forecasting and model accuracy.

1.2. Contribution. The main contributions of this paper are as follows:
This work proposes a Deep Learning Neural Networks prediction system enhanced with the best window size in sliding window algorithm for stably predicting domestic energy consumption in a residential building on 4.5 years of energy dataset

The proposed deep learning models are tuned to achieve high performance based on various hyperparameters

This study performs an extensive experimental evaluation of different variations of deep learning models and records the best RMSE value compared against the previous studies on the benchmark datasets

This work also focuses on analyzing the variables of interest that construct the energy consumption data followed by various model fitting functions for a precise explanation of the electric consumption dataset

The structure of the paper is organized as follows: Section 2 provides a brief introduction to deep learning recurrent neural network theory. Section 3 applies establishing deep recurrent neural network prediction models for domestic energy consumption in an actual residential house and analyzing various household appliances variables affecting the energy consumption forecasting, and performance measures and modification of hyperparameter for tuning the model performance will also be discussed in this section. In Section 4, the conclusions are presented.

\section{Introduction to Deep Learning Recurrent Neural Network Theory}

A specialized subset of machine learning is coined as "deep learning," which [51-58] overcomes the accuracy issues [59]. Deep learning architectures are composed of nonlinear computation at multiple levels, such as neural nets performed by many hidden layers [60] with more abstraction and complexity. The key feature of deep learning architectures is automated feature extraction and data scaling with improved high performance [61]. Several artificial intelligence applications are widely relying upon the foundation of Deep Neural Networks. It includes robotics, IoT sensor data, image, speech recognition, and many other breakthrough domains that have exploded, which use Deep Neural Networks [62-66].

In this paper, the effectiveness of deep learning methods is explored by executing domestic level forecasting in a residential building. The proposed methodology uses CNN and LSTM techniques. The proposed work introduces an LSTM and CNN model with an enhanced sliding window algorithm. Both deep learning techniques are established on a benchmark electricity consumption dataset for an individual residential building with daily time resolutions.

2.1. LSTM for Time Series. Principally, neural networks for time-series forecasting are commonly categorized in two variants, namely, (i) feedforward neural networks and (ii) deep learning recurrent neural networks. Feedforward neural networks only process spatial information and skip 
temporal information, whereas deep learning recurrent neural networks are capable of handling both sequential and temporal information as it is composed of fully connected neurons having look back features even on a large-scale dataset. Regardless of these benefits, the typical recurrent neural networks (RNN) deteriorate due to vanishing gradient issues while handling the long-term dependencies [67].

Hochreiter and Schmidhuber [68] proposed a new advancement based on connected subnets to overcome the RNN shortcoming known as Long Short-Term Memory. LSTM has the ability to remember observations at arbitrary time lags. Now, the question is what makes LSTM remember? In conventional RNN, each cell receives two inputs, output from the previous hidden state and values at the " $t$ " time. Apart from a hidden state, no past information exists to remember by the RNN. RNN cell representation is shown in Figure 1. The fundamental computations are provided in (1) and (2).

$$
\begin{aligned}
& h^{t}=\sigma\left[W_{x}^{h}+x^{t}+W_{h}^{h} * h^{t-1}+b^{h}\right], \\
& Z^{t}=\sigma\left[W_{Z}^{h} * h^{t}+b^{Z}\right],
\end{aligned}
$$

where $\sigma$ defines activation function, $W_{x}^{h}, W_{h}^{h}$, and $W_{Z}^{h}$ represent weight matrices of input, hidden, and output layer, and $b^{h}$ and $b^{Z}$ are bias at hidden and output layer, respectively.

A recurrently connected memory block uses memory cells with self-connections in addition to three multiplicative gates, namely, input gate $\left(i_{t}\right)$, input modulation gate $\left(\dot{c}_{t}\right)$, forgot gate $\left(f_{t}\right)$, and output gate $\left(O_{t}\right)$ in the hidden layer, enabling the model to store temporal state and to control the information flow in the network as directed by the associated activation function (ReLU, sigmoid, and tanh), thereby alleviating the vanishing of gradient problem. However, each gate plays a significant role.

Input gate is responsible for writing operations

Input modulation gate is responsible for the creation of a new cell value vector

Forgot gate, also known as remember vector, is responsible for taking the decision of which information is to be kept or which is to be forgotten

Output gate performs reading operations and decides which memory cell value passes to the next hidden state

Figure 2 shows the internal structure of LSTM. LSTM cell takes two inputs at " $\mathrm{t}$ " timestamp: (i) input at $t\left(X_{t}\right)$ and (ii) $\left(h_{t-1}\right)$ previous timestamps used to obtain the outcome. The working of each gate governs through a set of mathematical equations as presented in equations (3) to (7).

Cell state equation is as follows:

$$
C_{t}=f_{t} * C_{t-1}+i_{t} * \dot{c}_{\mathrm{t}}
$$

Input gate equation is as follows:

$$
i_{t}=\sigma\left(W_{i} *\left[h_{t-1}, x_{t}\right]+b_{i}\right) .
$$

Input modulation gate equation is as follows:

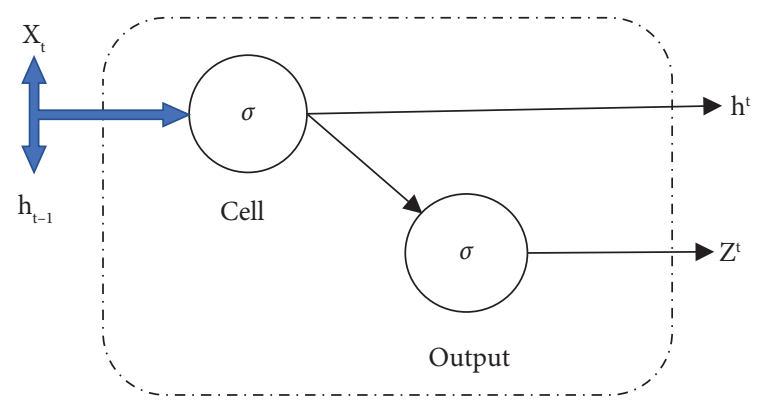

FIGURE 1: RNN cell representation.

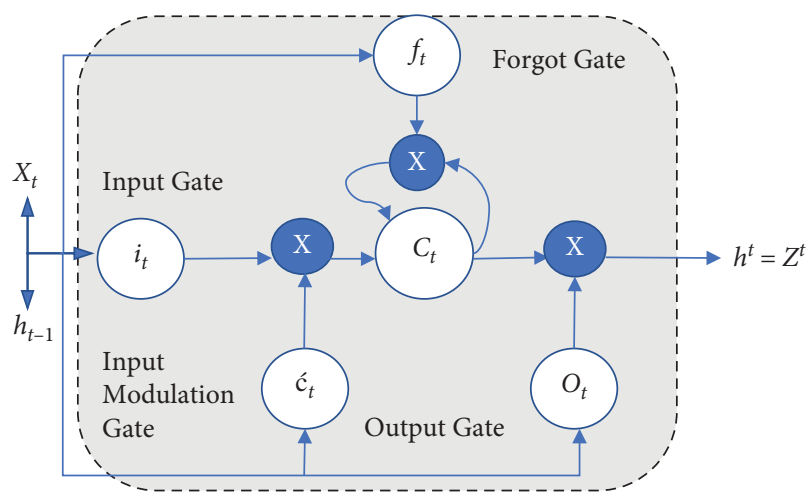

Figure 2: Long Short-Term Memory cell unit representation.

$$
\dot{c}_{\mathrm{t}}=\operatorname{Relu}\left(W_{\dot{c}} *\left[h_{t-1}, x_{t}\right]+b_{\dot{c}}\right) .
$$

Forgot gate equation is as follows:

$$
f_{t}=\sigma\left(W_{f} *\left[h_{t-1}, x_{t}\right]+b_{f}\right) .
$$

Output gate equation is as follows:

$$
O_{t}=\sigma\left(W_{o} *\left[h_{t-1}, x_{t}\right]+b_{o}\right),
$$

where $W_{i}, W_{\dot{c}} W_{f} W_{o}$ define the weight matrices for input and recurrent layer and $b_{i}, b_{\dot{c}}, b_{f}, b_{o}$ are the input and recurrent bias, respectively. Finally, the hidden state equation is defined for the output $\left(h_{t}\right)$ in equation (8), respectively.

Hidden state equation is as follows:

$$
h_{t}=O_{t} * \operatorname{Relu}\left(\dot{c}_{\mathrm{t}}\right) \text {. }
$$

However, during the training process, the initial parameter values, that is, weights and bias of the LSTM, are generated arbitrarily. In general, weights and bias of the neuron in the LSTM layers can be updated through the standard gradient descent method employed by the backpropagation algorithm, but the performance of gradient descent highly depends upon the selection of optimal hyperparameters, which improves the accuracy of timeseries problems.

2.2. CNN for Time Series. In general, there are a number of methodologies that perform analysis and prediction out of time-series data. However, these methodologies usually follow at least a two-step process: first, perform feature 
engineering and use some statistical algorithms or models to transform the time series as a vector of features; second, perform classification or regression tasks using various statistical or machine learning algorithms. On the other hand, CNN can be viewed as one frame architecture that incorporates the automatic extraction of features and modeling the time-series data. In this work, multivariate time-series data consists of " $n$ " length and " $k$ " width, where length defines time step count and width represents the total number of features. The CNN architecture viewed the input sequence as an image of " $n$ " pixels and each pixel with " $k$ " channels.

Unlike LSTM, CNN uses the current window and does not consider past information. The CNN consists of three layers, namely, input layer, multiple hidden layers, and output layer. The input layer receives extracted features as input, the output layer generates the prediction, and the hidden layer is further composed of three layers (convolution layer, ReLU layer, and pooling layer) and an activation function. Each sublayer of the hidden layer has a significant role in the prediction process: (i) Convolution layer uses convolution operation to the input sequence where convolution operation emulates the neurons' response, then processes the time-series data, and passes the outcome to the successor layer. (ii) ReLU layer is an activation function. (iii) Pooling layer is responsible for parameter reduction by reducing the space size for representation and thereby results in a reduction in computational cost. Equation (9) governs the $L$ th convolution layer outcome, and equation (10) provides the working of the pooling layer.

$$
\begin{aligned}
& y_{i j}=\sigma\left[\left(\sum_{m=1}^{M} W_{m, j}^{l} * X_{i+m-1, j}^{0}\right)+b_{j}^{l}\right] . \\
& P_{i j}^{l}=\frac{\text { Pooling Type }}{r \in R}\left(y_{i * T+r, j}^{l-1}\right),
\end{aligned}
$$

where $y_{i j}$ is the output vector, $b_{j}$ defines the bias, $W_{m, j}$ defines the weight metrics, $m$ defines the filter index value, $\sigma$ is the activation function, $T$ is the strides, $R$ is the pooling size (must be less than the size of input), and pooling type defines which pooling function is used out of three: maximum, average, and sum, respectively. However, this work applies the "max" pooling type for parameter reduction.

Consequently, CNN transforms the given input sequence layer by layer. However, gradient descent will be used to train the weights and biases of the neurons in the convolution layer or fully connected layer so that the prediction scores computed by the CNN remain consistent with the training set.

2.3. Sliding Window Algorithm and Window Size. Sliding window algorithm is used to construct samples with one step size where each sliding window includes previous time steps as an input, and it aims to predict the upcoming time step. Consider the input vector $X_{n}$ where subscript $n$ is the number of normalized 7-day units to be taken per window. The sliding window algorithm is able enough to handle multivariate datasets as an input to the model and perform data smoothing of the original data by reducing the model time complexity. In the sliding window algorithm, the window size is quite responsible for deciding the number of time steps that need to be considered in the past and obtaining the optimal model as the model will learn the energy data that is preprocessed for every defined fixed window length. Consequently, the static window size may limit the temporal modeling in the deep learning neural networks as the data defined by the window size are only being modeled and are inappropriate to handle long-term dependencies in the time-series data.

To the best of our knowledge, most of the researchers used fixed or static window size in the sliding window algorithm. By selecting the proper size of a sliding window, the model efficiency can also be improved. Hence, this work is finding the best $\mathrm{N}$ window size in order to yield the best model with the least Root Mean Square Error. To accomplish this objective, an independent function is created, which takes a minimum value and maximum value as the parameters in order to observe the best window length for " $\mathrm{N}$ " previous time steps for prediction and run the deep prediction models several times to process the time series in each window using sliding window approach. For every " $N$ " value, the window receives the input data from the beginning, and the time series is encoded into the vectors at the end of each window. Later, the obtained feature vectors are fed into the deep learning prediction models (i.e., LSTM and $\mathrm{CNN}$ ) to estimate the prediction error with its respective training labels. In summary, Algorithm 1 presents the pseudocode for the deep prediction models with an enhanced sliding window algorithm.

\subsection{Mathematical Formulation for Domestic Energy} Consumption. In this work, predicting a domestic energy consumption problem has a multivariable time-series dataset with "N" distinct attributes, which include electrical measuring parameters and sensors deployed at different sections of residential buildings. At each time step " $t$," electric energy consumed by the household appliances is recorded by the sensors attached to the submetering system and can be provided in the form of (11).

$$
X_{t}=\left(X_{t}^{1}, X_{t}^{2}, X_{t}^{3}, \ldots, X_{t}^{n}\right),
$$

where $X_{t}^{n}$ signifies the total number of variables constitute energy consumption data at the " $t$ " time step. Now, let us consider window size for input as this work includes a sliding window approach as follows:

$$
W S_{k}^{l}=\left(X_{k}, X_{k+1}, X_{k+2}, \ldots \ldots \ldots, X_{k+l-1}\right),
$$

where $l$ indicates the time-series length and training dataset with its labels represented as $l_{i}=X_{k+l}$. Therefore, models need to learn function " $f$," which provide the mapping of window onto its respective training label and can be designed by (13).

$$
l_{i}=\alpha\left(W S_{k}^{l}\right)
$$




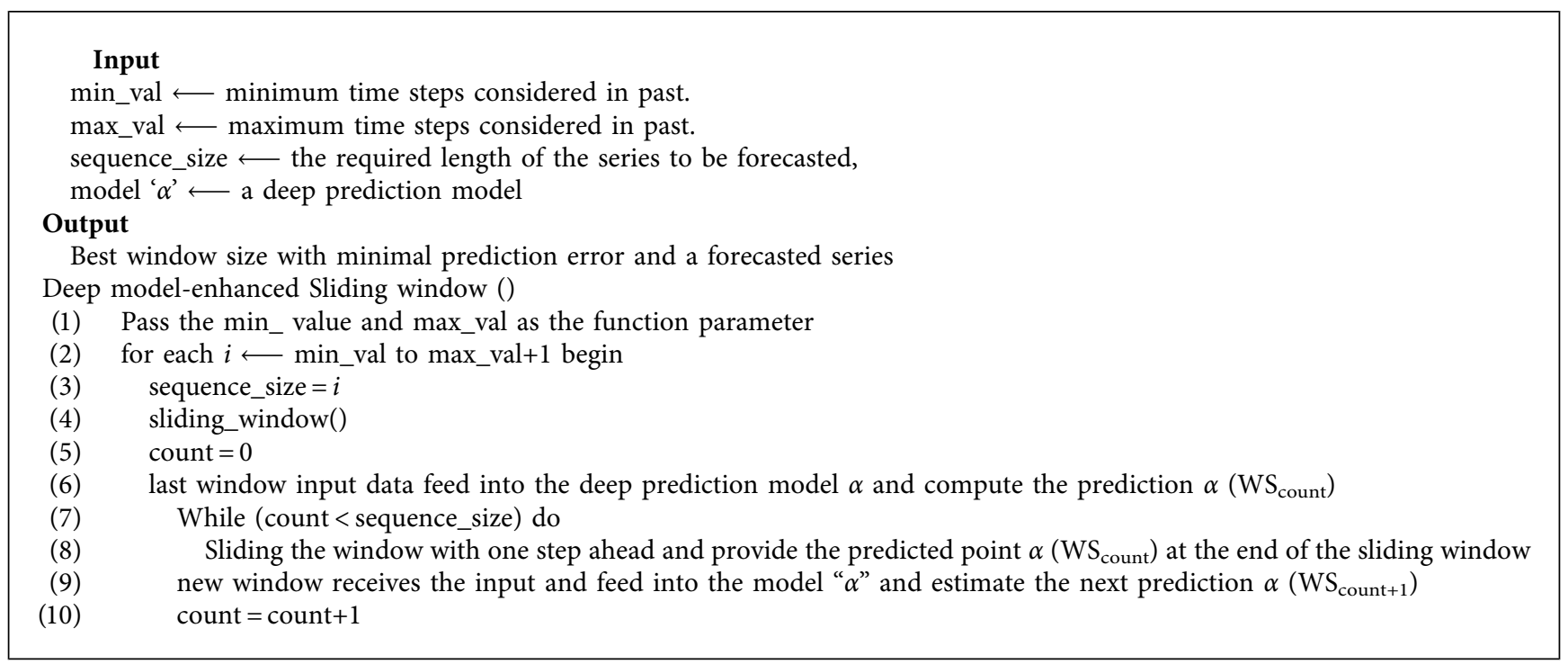

Algorithm 1: Deep prediction models with enhanced sliding window algorithm.

So that, given energy consumption prediction problem can be transformed into supervised learning problem and uses Root Mean Square Error for prediction error. Hence, the prediction formal derivation is shown in (14).

$$
\operatorname{Model}_{\Omega} \min \frac{1}{2} \sum_{i=1}^{N}\left(l_{i}-\alpha\left(W S_{k}^{l}\right)\right)^{2},
$$

where Model $_{\Omega}$ is the set of parameters for the model " $\alpha$ " and $N$ is training size. Model " $\alpha$ " is a deep learning model like LSTM and CNN.

\section{Establishing Deep Recurrent Neural Network Prediction Model for Domestic Energy Consumption}

In this section, detailed explanations on the proposed deep learning recurrent neural network prediction system enhanced with an improved sliding window algorithm for accurate energy consumption forecasting will be provided.

\subsection{Basic Electrical Energy Consumption of Residential} Building Dataset Information and Data Preprocessing. In this study, the electric power consumption dataset was collected from the UCI machine learning repository [69]. The dataset contains the readings collected from the monitored house, situated in the city of Sceaux near Paris in France. This multivariate dataset is composed of recorded observations covering the period of 4 years from December 2006 to November 2010. During preprocessing, a total of $1.25 \%$ of the rows contain missing values that were imputed with the median. Also, redundant records are tried to be removed so that they will not cause the learning model to be biased towards the more frequent records during the training process[70].

Different electrical measures and submetering information of interest that construct the energy consumption data of residential buildings are presented in Table 2. The active power is the actual energy consumed, and the unutilized energy in the power line is referred to as reactive power. Besides active power, the dataset contains a distribution of real power via the main circuit in different areas of the home, that is, kitchen, washing area, and temperature control systems via sensory submetering systems, whereas voltage represents the average voltage supplied and global intensity refers to the average current intensity.

The major electrical energy consumption of a residential building can be divided into three categories: cookhouse consumption, lighting and utility room consumption, and heating and cooling system power consumption, as shown in Figure 3. From Figure 3, it can be observed that the energy used by the cookhouse and utility room along with lighting accounts for less impact on global power consumption. On the other hand, energy consumed by heating and cooling system accounts for a major portion of residential's electrical energy consumption. The equipment of the cookware house includes microwave, oven, and dishwasher that are connected to submeter 1 , utility area includes lights, washing machine, drier, and refrigerator, which is connected to submeter 2, and heating and cooling units account for the water heater and air conditioner of the house concerned with the submetering 3 .

The results of various model fitting functions are summarized for a precise explanation of the electric consumption dataset, as mentioned in Table 3. These fitting functions will help in estimating and understanding the distributional properties for different energy variables that are conditional on other variables and summarize the relationship among variables.

3.2. Applying LSTM and CNN Deep Learning Model. The proposed deep learning recurrent neural network protection system was implemented on the Anaconda Navigator using Python version 3.7 on an Intel Core i5 $1.60 \mathrm{GHz}$ processor 
TABLE 2: Different electrical measures and submetering information of interest that construct the energy consumption data.

\begin{tabular}{lcc}
\hline Variables & Description & Measuring units \\
\hline Global active power & Total active power & Kilowatts \\
Global reactive power & Total reactive power & Kilowatts \\
Voltage & Average voltage & Volts \\
Global intensity & Average current intensity & Ampere \\
Submetering 1 & Active energy corresponds to the kitchen & Watt-hours of active energy \\
Submetering 2 & Active energy corresponds to the laundry & Watt-hours of active energy \\
Submetering 3 & Active energy corresponds to the cooling and heating appliances & Watt-hours of active energy \\
\hline
\end{tabular}

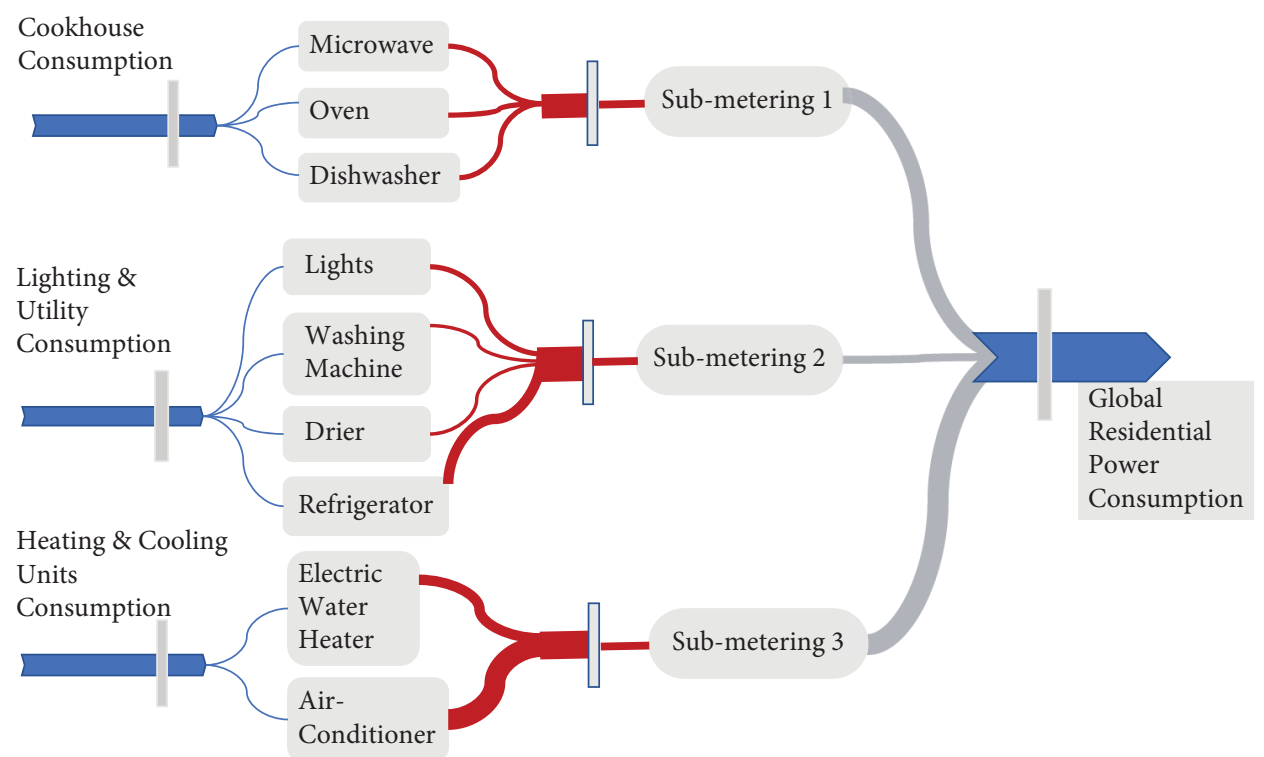

FIGURE 3: Structure of electrical energy consumption of residential buildings.

TABle 3: Various model fitting functions for a precise explanation of the electric consumption dataset.

\begin{tabular}{lccccccc}
\hline Fitting function & Min & 1st quantile & Median & Mean & 3rd quantile & Max & Standard deviation \\
\hline Global active power & 0.076 & 0.308 & 0.602 & 1.092 & 1.528 & 11.122 & 1.055 \\
Global reactive power & 0.000 & 0.048 & 0.100 & 0.124 & 0.194 & 1.390 & 0.113 \\
Voltage & 223.2 & 239.0 & 241.0 & 240.8 & 242.9 & 254.2 & 3.239 \\
Global intensity & 0.200 & 1.400 & 2.600 & 4.628 & 6.400 & 48.400 & 4.435 \\
Submetering 1 & 0.000 & 0.000 & 0.000 & 1.122 & 0.000 & 88.000 & 6.139 \\
Submetering 2 & 0.000 & 0.000 & 0.000 & 1.299 & 1.000 & 80.000 & 5.794 \\
Submetering 3 & 0.000 & 0.000 & 1.000 & 6.458 & 17.00 & 31.000 & 8.436 \\
\hline
\end{tabular}

with $8 \mathrm{~GB}$ ram on Windows 10 operating system. The whole experimental setup is divided into 3 steps, namely, (i) data preprocessing, (ii) data partition into training and testing dataset, and (iii) modification of hyperparameters.

3.2.1. Data Preprocessing. A preliminary and inescapable phase in forecasting applications is used to reshape the obtained domestic electric power consumption dataset, from Section 3.1, into a proper format that can be fed into the learning model and aid in the convergence of the learning model. In this work, the $\mathrm{z}$-score normalization technique was applied to the input features, thereby providing data transformation in such a way where the mean value equals zero and the standard deviation is 1 for the transformed data in order to prevent inaccurate predictions because of the presence of high-frequency components in energy consumption dataset. The mathematical formulation for $\mathrm{z}$-score data transformation is shown in (15).

$$
t_{i}=\frac{\left(t_{i}-\overline{t_{i}}\right)}{S_{i}} ; \quad \forall i=(1,2,3, \ldots, n),
$$

where $\overline{t_{i}}$ is the mean value of data samples, $S_{i}$ defines the standard deviation of data samples, and $n$ is the total number of data samples in the dataset. A significant highlight to be 
noted here is that prediction errors are recorded in their original scale in terms of various performance metrics, as discussed in Section 3.3.

3.2.2. Data Partition. In the data partition step, the considered domestic energy consumption dataset is partitioned in order to generate training and testing data samples to reduce the learning model complexity and thereby optimize the accuracy. The dataset consists of 2075259 observations. For experiment, original energy data sampled at 1-minute granularity was later downsampled at the daily interval for meaningful analytics. Further, the sliding window approach was used for the short-term prediction of energy demand for a day. Initially, the sliding window of sequence size of seven points per window was considered, which is equivalent to 7 days in the past, in order to forecast the next point that is 24 hours or a day in the future.

Further to study the impact of window size on prediction error, sliding window approach enhanced with window size function which takes several ranges and tries to obtain the best $\mathrm{N}$ window size means the total number of days in the past that yields an optimal model with minimal prediction error as shown and discussed in Section 3.4. The training data samples for short-term prediction consist of energy consumption of a residential building for the period of three years using the aforementioned window size of 7 data points, and the rest of the one year of the energy dataset is held out purely for testing purpose.

3.2.3. Modification of Hyperparameter. In general, hyperparameter is the model property governing the training process [70]. Hyperparameter covers two types of variables: one determines the model structure, and the other determines how the model is trained. Optimizing the hyperparameter has a significant impact on the performance of the model.

In this work, optimizer, activation function, neuron count in each layer, number of LSTM layers, kernel size, and kernel number (in case of CNN) followed by early stopping and model checkpoint hyperparameters were tuned to check out how these affect the model performance. Besides that, the dropout technique was used to perform regularization to avoid overfitting and generalization errors.

3.3. Performance Measures. The statistical measures are assessed to determine which model is suitable to amplify the goodness of fit using the classical model or hybridization of the model. In our work, the following set of statistical measures are selected to evaluate the prediction performance of the deep learning recurrent neural network prediction system over three states of the art of residential energy consumption, namely, Mean Square Error (MSE), Root Mean Square Error (RMSE), and coefficient of determination $\left(R^{2}\right)$. The statistical metrics are defined through equations (16)-(18), respectively.

$$
\begin{aligned}
\text { MSE } & =\frac{1}{N} \sum_{i=1}^{N}\left(y_{i}-\hat{y}_{i}\right)^{2} . \\
\text { RMSE } & =\sqrt{\frac{1}{N} \sum_{i=1}^{N} \in \text { Test }}\left(y_{i}-\hat{y}_{i}\right)^{2} . \\
R^{2} & =1-\frac{\sum_{i=1}^{N}\left(y_{i}-y_{i}^{\text {pred }}\right)^{2}}{\sum_{i=1}^{N}\left(y_{i}-y^{\text {mean }}\right)^{2}},
\end{aligned}
$$

where at time step " $i$," $y_{i}$ is the observed energy consumption, $\hat{y}_{i}$ is the predicted energy consumption, and " $N$ " is the data point count in the test dataset.

\subsection{Results of Deep Learning Models for Prediction of Domestic} Energy Consumption. In this section, various experimental results are discussed for all variations of models implemented using LSTM and CNN enhanced with a sliding window approach. Also, a comparative analysis was performed to demonstrate the performance of the proposed model over competitive benchmarks using a household electricity energy consumption dataset. The main focus of this work is to implement a robust residential electricity energy consumption prediction system with minimal prediction error and high accuracy. The proposed model integrates both deep learning models, that is, LSTM and CNN, with the improved version of the sliding window algorithm, which is capable of identifying the best $n$ window size and predicting the short-term energy consumption in a residential building located in the city of France. The parameter setting of the deep learning recurrent neural network prediction system enhanced with a sliding window algorithm is provided in Table 4. The LSTM and CNN models were implemented with TensorFlow at the backend, and the scikit-learn package was used for the implementation of other approaches in Python programming language on the Keras framework.

3.4.1. Performance Comparison of All Model Variations for LSTM and CNN Models. A primary strategy was to conduct the experiments to find out the optimal LSTM and CNN network structure, that is, to tune various hyperparameters, as discussed in Section 3.2.3, while training the model using TensorFlow for predicting the household electric energy consumption scenario.

Tables 5 and 6 provide the details of hyperparameter tuning to observe how they affect the performance of the prediction system for predicting domestic energy consumption scenarios in terms of Mean Square Error and Root Mean Square Error. The above tables show the LSTM architecture with "Adam" optimizer (activation function $=$ "ReLU," dropout $=0.2$, recurrent dropout $=0.2$, 
TABLE 4: Parameter setting of the proposed prediction system.

\begin{tabular}{lc}
\hline Parameters & Range \\
\hline Learning rate & $(0.01,0.001)$ \\
Decay & $(1 e-6)$ \\
Momentum & $(0.9)$ \\
Beta_1 & $(0.9)$ \\
Beta_2 & $(0.999)$ \\
Epsilon & $($ None) \\
Batch size & 128 \\
Epoch & 100 \\
\hline
\end{tabular}

TABLE 5: Evaluation of different variations of LSTM model with sliding window algorithm.

\begin{tabular}{lccc}
\hline Optimizer & Neuron count and number of LSTM layers & Activation function & Estimated Root Mean Square Error (RMSE) \\
\hline SGD & $128,128,128$ & ReLU, ReLU, sigmoid & 0.0722 \\
Adam & $\mathbf{1 2 8 , 6 4}$ & ReLU, ReLU & $\mathbf{0 . 0 6 9 3}$ \\
Adam & 128 & ReLU & 0.0764 \\
Adam & 32 & ReLU & 0.0877 \\
\hline
\end{tabular}

TABLE 6: Comparison of various model variations implemented by CNN with sliding window algorithm.

\begin{tabular}{lccc}
\hline Optimizer used & Kernel size and kernel number & Activation function and layers & Estimated Root Mean Square Error (RMSE) \\
\hline Adagrad & $(1,3),(1,1)$ and 64,128 & ReLU and ReLU & 0.1330 \\
Adamax & $(1,3),(1,1)$ and 128,264 & ReLU and ReLU & 0.1201 \\
Adam & $(1,3),(1,1)$ and 64, 128 & ReLU and ReLU & 0.1183 \\
Adamax & $(\mathbf{1 , 3}),(\mathbf{1}, \mathbf{1})$ and $\mathbf{6 4}, \mathbf{1 2 8}$ & ReLU and ReLU & $\mathbf{0 . 0 8 3 6}$ \\
Adamax & $(1,3),(1,3)$ and 64, 128 & ReLU and ReLU & 0.1110 \\
\hline
\end{tabular}

neuron count $=(128,64)$, and LSTM layer count $=2)$ performing optimally for short-term prediction and CNN architecture with "Adamax" optimizer (activation function = "ReLU," kernel size $=(1,3), \quad(1,1)$, kernel number $=64,128$, strides $=(1,1)$, pool size $=(1,1)$, and pooling type $=$ "max") executing in an optimal manner for short-term prediction. Figure 4 and Figure 5 present single household electric power consumption prediction results of proposed deep learning neural network prediction system for short-term scenario, that is, one day ahead prediction of global power consumption.

3.4.2. Optimal Window Size. This work confirmed the impact of changes in window size on deep learning models. Figures 6 and 7 present the optimal $\mathrm{N}$ window size graph for both LSTM and CNN models. The window size function takes several values to identify the best size for the sliding window as the fixed-size window length may limit the temporal modeling and also affect the model performance by increasing the forecasting error. Hence, this study analyzed and evaluated the proposed deep learning prediction system to be robust in the matter of dynamic window size and then compared the RMSE for the respective window size.

From the above figures, it was clear that LSTM with an improved sliding window algorithm gives better prediction performance with a window size of 7,12 , and 14, which means that these are the days that can be considered in the past sequence to obtain the minimal forecasting error. On the other hand, CNN with an improved sliding window

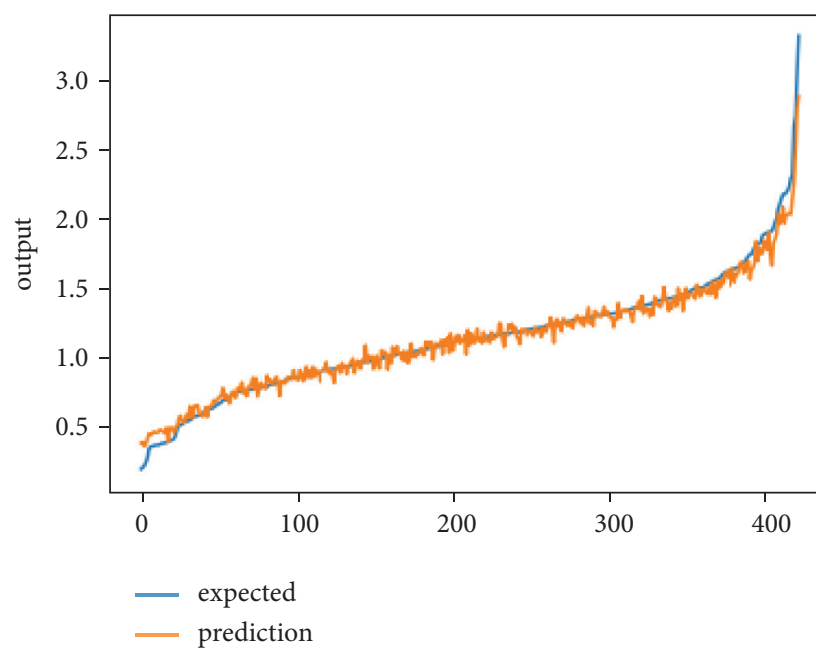

FIGURE 4: Regression lift chart showing original and prediction values of household energy consumption using the LSTM model.

approach provided minimal prediction error with the window size for 4,5 , and 8 , respectively.

3.4.3. Comparative Analysis with Competitive Benchmark. Further, a comparative study of the proposed work is performed with contrast benchmark models that use the considered household electricity consumption data. The prediction performance of the contrast model for the test 


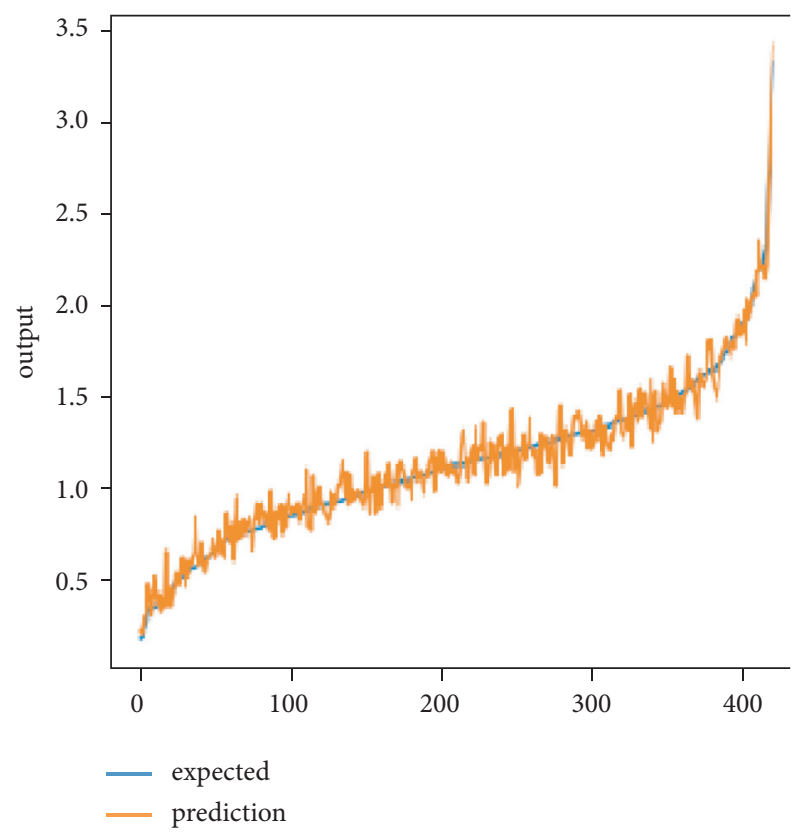

FIGURE 5: Regression lift chart showing original and prediction values of household energy consumption using CNN model.

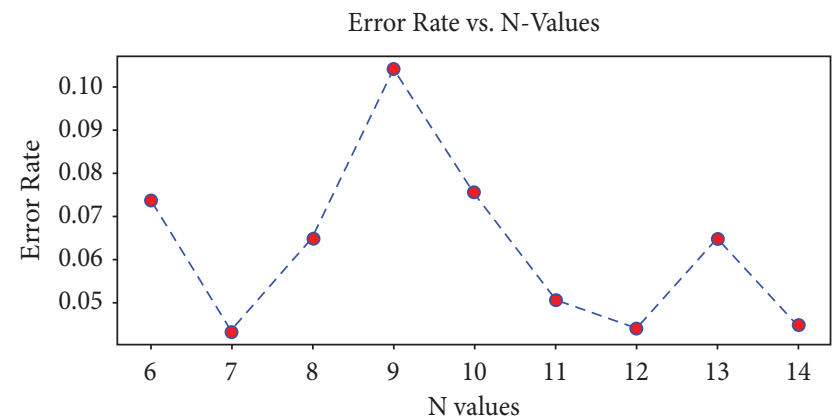

Figure 6: Best $\mathrm{N}$ window size graph for LSTM model.

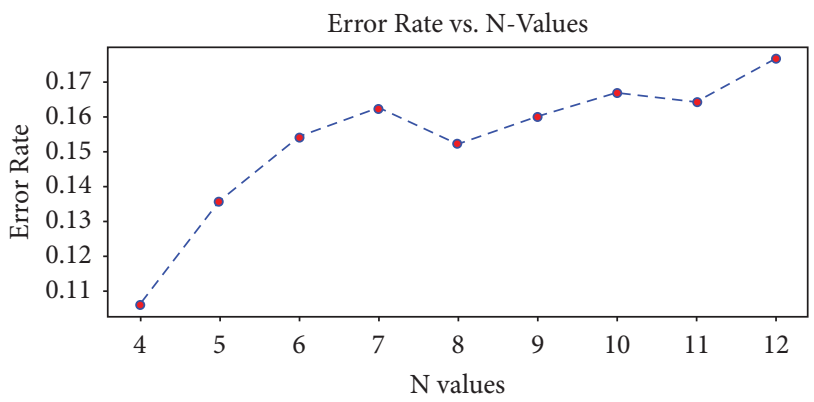

Figure 7: Best $\mathrm{N}$ window size graph for CNN model.

dataset for daily time resolution is summarized in Table 7 . Kim and Cho [16] stably predicted the total power consumption for a residential house using a hybrid approach by combining CNN and LSTM and applied a sliding window algorithm that enables the hybrid model to learn the input data. They fixed the window size to predict the minutely household consumption, but for the rest of the time
TABLE 7: Prediction performance of contrast model.

\begin{tabular}{lcccc}
\hline Method & Time resolution & MSE & RMSE & $R^{2}$ \\
\hline ARMA [25] & Daily & - & 0.34 & \\
CNN-LSTM [16] & Daily & 0.1037 & 0.3221 & \\
LSTM & Daily & 0.0048 & 0.0693 & 0.9679 \\
CNN & Daily & 0.0069 & 0.0836 & 0.9622 \\
\hline
\end{tabular}

resolutions like hourly, daily, and weekly, window size remains unclear. Chujai et al. [25] analyzed the energy consumption based on autoregressive models, that is, ARIMA and ARMA, using a benchmark dataset. They figured out forecasting errors for fixed and random window sizes. By comparing the error metrics, this work confirmed that the proposed deep learning recurrent neural network prediction system with an improved sliding window algorithm outperforms the considered contrasting benchmarks and also figured out the optimal window size for which the model achieved a minimal prediction error.

\section{Conclusion}

In this research work, an establishment of a residential energy prediction system is presented using the deep learning recurrent neural network; that is, LSTM and CNN models are used with an improved sliding window-based approach to establish a robust and accurate prediction of energy consumption in a residential building. This work employs a new feature in the sliding window algorithm of finding the optimal $N$ value for window size that is the sequence length of data points to be considered in the past. This improvement does not limit the temporal modeling and yields an optimal model with minimal forecasting error.

A case study on individual household electric power consumption data collected from a residential building located in France to predict the total active power consumption for a day with daily time resolution, a short-term scenario, was presented. In this course of the evaluation, the impact of hyperparameter tuning was analyzed for the shortterm scenario. Furthermore, the proposed deep learning prediction model outperforms benchmark contrast models in terms of different quality metrics like MSE and RMSE.

Although the proposed approach shows better prediction results, in the modeling process, the potential challenges are observed, which also provide a future directive to this study, as follows:

Automating the hypertuning using some evolutionary approach

Introducing additional features such as building parameters and occupancy parameters

Impact of different time resolutions on model performance

\section{Data Availability}

The multivariate time series energy data used to support the findings of this study are available from the corresponding author upon request. 


\section{Conflicts of Interest}

The authors declare no conflicts of interest.

\section{References}

[1] International Energy Agency, "Tracking clean energy progress 2016," 2016, http://www.iea.org/publications/ freepublications/publication/ TrackingCleanEnergyProgress2016.pdf.

[2] W. Yu, B. Li, Y. Lei, and M. Liu, "Analysis of A residential building energy consumption demand model," Energies, vol. 4, no. 3, pp. 475-487, 2011.

[3] S. Naji, A. Keivani, S. Shamshirband et al., "Estimating building energy consumption using extreme learning machine method," Energy, vol. 97, pp. 506-516, 2016.

[4] K. Amasyali and N. M. El-Gohary, "A review of data-driven building energy consumption prediction studies," Renewable and Sustainable Energy Reviews, vol. 81, pp. 1192-1205, 2018.

[5] M. M. Rathore, A. Paul, W.-H. Hong, H. Seo, I. Awan, and S. Saeed, "Exploiting IoT and big data analytics: defining smart digital city using real-time urban data," Sustainable Cities and Society, vol. 40, pp. 600-610, 2018.

[6] J. G. Jetcheva, M. Majidpour, and W.-P. Chen, "Neural network model ensembles for building-level electricity load forecasts," Energy and Buildings, vol. 84, pp. 214-223, 2014.

[7] X. Liu, C. Marnay, W. Feng, N. Zhou, and N. Karali, “A review of the american recovery and reinvestment act smart grid projects and their implications for China," 2017, https://china. lbl.gov/publications/review-american-recovery-and.

[8] D. R. Obey, "Text-H.R.1-111th Congress (2009-2010): american recovery and reinvestment act of 2009," 2009, https://www.congress.gov/bill/111thcongress/house-bill/1/ text.

[9] Smart Metering Deployment in the European Union|JRC Smart Electricity Systems and Interoperability. (n.d.). Retrieved from https://ses.jrc.ec.europa.eu/smartmeteringdeployment-european-union.

[10] U.S. Energy Information Administration (Eia), "How many smart meters are installed in the United States, and who has them?," 2018, https://www.eia.gov/tools/faqs/faq.php? $\mathrm{id}=108 \& \mathrm{t}=3$.

[11] C. Beckel, L. Sadamori, T. Staake, and S. Santini, "Revealing household characteristics from smart meter data," Energy, vol. 78, pp. 397-410, 2014.

[12] F. Haldi and D. Robinson, "The impact of occupants' behaviour on building energy demand," Journal of Building Performance Simulation, vol. 4, no. 4, pp. 323-338, 2011.

[13] S.-H. Liao, P.-H. Chu, and P.-Y. Hsiao, "Data mining techniques and applications - a decade review from 2000 to 2011," Expert Systems with Applications, vol. 39, no. 12, pp. 1130311311, 2012.

[14] H.-X. Zhao and F. Magoulès, "A review on the prediction of building energy consumption," Renewable and Sustainable Energy Reviews, vol. 16, no. 6, pp. 3586-3592, 2012.

[15] M. A. Mat Daut, M. Y. Hassan, H. Abdullah, H. A. Rahman, M. P. Abdullah, and F. Hussin, "Building electrical energy consumption forecasting analysis using conventional and artificial intelligence methods: a review," Renewable and Sustainable Energy Reviews, vol. 70, pp. 1108-1118, 2017.

[16] T.-Y. Kim and S.-B. Cho, "Predicting residential energy consumption using CNN-LSTM neural networks," Energy, vol. 182, pp. 72-81, 2019.
[17] G. Wang, W. Li, M. A. Zuluaga et al., "Interactive medical image segmentation using deep learning with image-specific fine tuning," IEEE Transactions on Medical Imaging, vol. 37, no. 7, pp. 1562-1573, 2018.

[18] A. Ullah, J. Ahmad, K. Muhammad, M. Sajjad, and S. W. Baik, "Action recognition in video sequences using deep Bi-directional LSTM with CNN features," IEEE Access, vol. 6, pp. 1155-1166, 2018.

[19] E. Protopapadakis, A. Voulodimos, and A. Doulamis, “Automatic crack detection for tunnel inspection using deep learning and heuristic image post-processing," Applied Intelligence, vol. 49, pp. 2793-2806, 2019.

[20] R. Nawaratne, D. Alahakoon, D. D. Silva, and X. Yu, "Spatiotemporal anomaly detection using deep learning for realtime video surveillance," IEEE Transactions on Industrial Informatics, vol. 16, no. 1, pp. 393-402, 2020.

[21] R. Haeb-Umbach, "Speech processing for digital home assistants: combining signal processing with deep-learning techniques," IEEE Signal Processing Magazine, vol. 36, no. 6, pp. 111-124, 2019.

[22] J. Zhao, X. Mao, and L. Chen, "Speech emotion recognition using deep 1D \& 2D CNN LSTM networks," Biomedical Signal Processing and Control, vol. 47, pp. 312-323, 2019.

[23] N. I. Widiastuti, "Convolution neural network for text mining and natural language processing," IOP Conference Series: Materials Science and Engineering, vol. 662, no. 5, 2019.

[24] J. Wang, L. C. Yu, K. R. Lai, and X. Zhang, "Dimensional sentiment analysis using A regional CNN-LSTM model in the proceedings of the," Annual Meeting of the Association for Computational Linguistics, vol. 2, pp. 225-230, 2016.

[25] P. Chujai, N. Kerdprasop, and K. Kerdprasop, "Time series analysis of household electric consumption with ARIMA and ARMA models," in Proceedings of the International MultiConference of Engineers and Computer Scientists, Hong Kong, March 2013.

[26] S. Ozturk and F. Ozturk, "Forecasting energy consumption of Turkey by arima model in Journal of asian scientific research," Asian Economic and Social Society, vol. 8, no. 2, pp. 52-60, 2018.

[27] T. E. Dikaios, "Forecasting residential electricity consumption in Greece using monthly and quarterly data," Energy Economics, vol. 14, no. 3, pp. 226-232, 1992.

[28] S. Katara, A. Faisal, and G. M. Engmann, "A time series analysis of electricity demand in tamale, Ghana," International Journal of Statistics and Applications, vol. 4, pp. 269275, 2014.

[29] J. Zhuang, Y. Chen, X. Shi, and D. Wei, "Building cooling load prediction based on time series method and neural networks," International Journal of Grid and Distributed Computing, vol. 8, pp. 105-114, 2015.

[30] H. Nie, G. Liu, X. Liu, and Y. Wang, "Hybrid of ARIMA and SVM for short-term load forecasting," Energy Procedia, vol. 16, pp. 1455-1460, 2011.

[31] B. Wang, N. Tai, H. Zhai, J. Ye, J. Zhu, and L. Qi, "A new ARMAX model based on evolutionary algorithm and particle swarm optimization for short-term load forecasting," Electric Power Systems Research, vol. 78, pp. 1679-1685, 2008.

[32] S. Abosedra and B. Campus, "Demand for electricity in Lebanon," International Business \& Economics Research Journal, vol. 8, 2009.

[33] N. Fumo and M. Biswas, "Regression analysis for prediction of residential energy consumption," Renewable and Sustainable Energy Reviews, vol. 47, pp. 332-343, 2015. 
[34] J. S. Chou and D. K. Bui, "Modeling heating and cooling loads by artificial intelligence for energy-efficient building design," Energy and Buildings, vol. 82, pp. 437-446, 2014.

[35] K. P. Amber, M. W. Aslam, and S. K. Hussain, "Electricity consumption forecasting models for administration buildings of the UK higher education sector," Energy Building, vol. 90, pp. 127-136, 2015.

[36] Y. Ji and P. Xu, "A bottom-up and procedural calibration method for building energy simulation models based on hourly electricity submetering data," Energy, vol. 93, no. 2, pp. 2337-2350, 2015.

[37] M. R. Braun, H. Altan, and S. B. M. Beck, "Using regression analysis to predict the future energy consumption of A supermarket in the UK," Applied Energy, vol. 130, pp. 305-313, 2014.

[38] A. Gelman, J. Carlin, H. Stern, D. Dunson, A. Vehtari, and D. Rubin, Bayesian Data AnalysisChapman and Hall/CRC, New York, 2013.

[39] S. L. Wong, K. K. W. Wan, and T. N. T. Lam, "Artificial neural networks for energy analysis of office buildings with daylighting," Applied Energy, vol. 87, no. 2, pp. 551-557, 2010.

[40] R. E. Edwards, J. New, and L. E. Parker, "Predicting future hourly residential electrical consumption: a machine learning case study," Energy and Buildings, vol. 49, pp. 591-603, 2012.

[41] M. A. R. Biswas, M. D. Robinson, and N. Fumo, "Prediction of residential building energy consumption: a neural network approach," Energy, vol. 117, pp. 84-92, 2016.

[42] M. W. Ahmad, M. Mourshed, and Y. Rezgui, "Trees vs neurons: Comparison between random forest and ANN for high-resolution prediction of building energy consumption," Energy and Buildings, vol. 147, pp. 77-89, 2017.

[43] M. Barman, N. B. Dev Choudhury, and S. Sutradhar, "A regional hybrid Goa-SVM model based on similar day approach for short-term load forecasting," Energy, vol. 145, pp. 710-720, 2018.

[44] W.-C. Hong, "Electric load forecasting by support vector model," Applied Mathematical Modelling, vol. 33, pp. 24442454, 2009.

[45] M. Božic, M. Stojanovi, and Z. Stajic, "Short-term electric load forecasting using least square support vector machines," Automatic Control and Robotics, vol. 9, no. 1, pp. 141-150, 2010.

[46] D. Liu, Q. Chen, and K. Mori, “Time series forecasting method of building energy consumption using support vector regression," in Proceedings of the IEEE International Conference On Information And Automation, pp. 1628-1632, Lijiang, China, August 2015.

[47] L. Qiong, R. Peng, and M. Qinglin, "Prediction model of annual energy consumption of residential buildings," in Proceedings of the International Conference on Advances in Energy Engineering, pp. 223-226, Beijing, June 2010.

[48] Y. Fu, Z. Li, H. Zhang, and P. Xu, "Using support vector machine to predict next day electricity load of public buildings with sub-metering devices," Procedia Engineering, vol. 121, pp. 1016-1022, 2021.

[49] C. Deb, F. Zhang, J. Yang, S. E. Lee, and K. W. Shah, "A review on time series forecasting techniques for building energy consumption," Renewable and Sustainable Energy Reviews, vol. 74, pp. 902-924, 2017.

[50] M. Bourdeau, X. G. Zhai, E. Nefzaoui, X. Guo, and P. Chatellier, "Modeling and forecasting building energy consumption: a review of data-driven techniques," Sustainable Cities and Society, vol. 48, pp. 1015-1033, 2019.
[51] N. Beliaeva, A. Petrochenkov, and K. Bade, "Data set analysis of electric power consumption," International Multi-disciplinary Journal, vol. 61, pp. 2482-2487, 2013.

[52] A. Bogomolov, B. Lepri, R. Larcher, F. Antonelli, F. Pianesi, and A. Pentland, "Energy consumption prediction using people dynamics derived from cellular network data," EPJ Data Science, vol. 5, no. 1, 2016.

[53] M. Shao, X. Wang, Z. Bu, X. Chen, and Y. Wang, "Prediction of energy consumption in hotel buildings via support vector machines," Sustainable Cities and Society, vol. 57, pp. 102-128, 2020.

[54] M. Chammas, A. Makhoul, and J. Demerjian, "An efficient data model for energy prediction using wireless sensors," Computers \& Electrical Engineering, vol. 76, pp. 249-257, 2019.

[55] L. Tang, X. Wang, X. Wang, C. Shao, S. Liu, and L. Tian, "Long-term electricity consumption forecasting based on expert prediction and fuzzy bayesian theory," Energy, vol. 167, pp. 1144-1154, 2019.

[56] Z. Wang, Y. Wang, and R. S. Srinivasan, "A novel ensemble learning approach to support building energy use prediction," Energy and Buildings, vol. 159, pp. 109-122, 2018.

[57] D. L. Marino, K. Amarasinghe, and M. Manic, "Building energy load forecasting using deep neural networks," in Proceedings of the IECON 2016 - 42nd Annual Conference of the IEEE Industrial Electronics Society, Florence, Italy, October 2016.

[58] E. Mocanu, P. H. Nguyen, M. Gibescu, and W. L. Kling, "Deep learning for estimating building energy consumption," Sustainable Energy, Grids and Networks, vol. 6, pp. 91-99, 2016.

[59] A. Wolfewicz, "Deep learning vs machine learning-what's the difference?," 2020, https://www.levity.ai/blog/differencemachine-learning-deep-learning.

[60] A. Krizhevsk, I. Sutskever, and G. Hinton, "Image net classification with deep convolutional neural networks," Advances in Neural Information Processing Systems, vol. 25, pp. 1106-1114, 2012.

[61] X. Ma, "A survey on deep learning empowered IoT applications,” IEEE Access, vol. 7, pp. 181721-181732, 2019.

[62] M. Pfeiffer, M. Schaeuble, J. Niet, R. Siegwart, and C. Cadena, "From perception to decision: a data-driven approach to endto-end motion planning for autonomous ground robot," in Proceedings of the IEEE International Conference on Robotics and Automation (ICRA), pp. 1527-1533, Singapore, June 2017.

[63] A. Krizhevsky, I. Sutskever, and G. E. Hinton, "Image net classification with deep convolutional neural networks," Communications of the ACM, vol. 60, no. 6, pp. 84-90, 2017.

[64] O. Russakovsky, J. Deng, and H. Su, "Image net large scale visual recognition challenge," International Journal of Computer Vision, vol. 115, no. 3, pp. 211-252, 2015.

[65] L. Deng, J. Li, J. T. Huang, and K. Yao, "Recent advances in deep learning for speech research at microsoft," in Proceedings of the IEEE International Conference on Acoustics, Speech and Signal Processing (ICASSP), pp. 8604-8608, Vancouver, Canada, May 2013.

[66] D. Tomar, P. Tomar, and G. Kaur, "Deep learning in big data and the internet of things," Information, Communication and Computing Technology (ICICCT), vol. 835, pp. 70-81, 2019.

[67] M. Wielgosz, A. Skoczeń, and M. Mertik, "Using LSTM recurrent neural networks for monitoring the LHC superconducting magnets," Nuclear Instruments and Methods in Physics Research Section A: Accelerators, Spectrometers, Detectors and Associated Equipment, vol. 867, pp. 40-50, 2017. 
[68] S. Hochreiter and J. Schmidhuber, "Long short-term memory,” Neural Computation, vol. 9, pp. 1735-1780, 1997.

[69] G. Hebrail and A. Berard, "Individual household electric power consumption data set. UCI Machine Learning Repository," 2012, https://archive.ics.uci.edu/ml/index.php.

[70] Prabhu, "Understanding hyperparameters and its optimisation techniques," 2018, https://towardsdatascience.com/ understanding-hyperparameters-and-its-optimisationtechniques-f0debba07568. 